\title{
Paroxysmal and non-paroxysmal atrial fibrillation: does the arrhythmia type influence thromboembolic risk?
}

\author{
Vitlianova Katerina Dimitrova ${ }^{1}$, Negreva Mariya Negrinova ${ }^{2, *}$ and Madjova Christiana Rosenova ${ }^{3}$ \\ ${ }^{1}$ Clinic of Cardiology, Second City Hospital of Sofia, Sofia, Bulgaria; address: 120 Hr Botev str., Sofia 1202, Bulgaria. \\ ${ }^{2}$ Department of Cardiology, Medical University of Varna, First clinic of cardiology, University Hospital "St. Marina"-Varna, \\ Varna, Bulgaria. \\ ${ }^{3}$ Department of Conservative dentistry and Oral Pathology, Faculty of Dental Medicine, Medical University-Varna, Varna, \\ Bulgaria.
}

Publication history: Received on 15 April 2020; revised on 20 April 2020; accepted on 22 April 2020

Article DOI: https://doi.org/10.30574/wjarr.2020.6.1.0104

\begin{abstract}
Atrial fibrillation (AF) is the most commonly diagnosed rhythmic disorder in clinical practice. Thromboembolic events are the main problem caused by the disease. Incidence, morbidity, and affected population are significantly different for the different types of AF. This quite logically raises the question of possible differences in the thromboembolic potential of paroxysmal and non-paroxysmal (persistent and permanent) AF. However, most studies address the problem for the entire population of AF patients. Those who distinguish between the paroxysmal and the non-paroxysmal type produce contradictory results. There is no consensus on the thromboembolic risk depending on the disease duration (paroxysmal/non-paroxysmal AF). According to current clinical guidelines, anticoagulant therapy is independent of AF type. The fact that AF has a dynamic nature and its paroxysmal form often progresses to non-paroxysmal cannot be overlooked. Further studies in search of the embologenic threshold of AF and biomarkers for its diagnosis are necessary for optimization of anticoagulant prophylaxis.
\end{abstract}

Keywords: Paroxysmal/non-paroxysmal atrial fibrillation; Embolic risk; Anticoagulation

\section{Introduction}

Atrial fibrillation is an important clinical and social problem. Over the past two decades, the disease has become one of the leading causes of worsening public health indicators and raising health care costs in developed countries in Europe and the USA. AF alone does not pose an immediate threat to the lives of affected patients. However, it has significant adverse effects on myocardial anatomy, hemodynamics and coagulation, which predetermine the observed complications. The rhythmic disturbance manifestation is associated with frequent hospitalizations, cognitive dysfunction and impaired work capacity [1]. The major clinical problem caused by the disease are thromboembolic events and, most often as a consequence, mortality. As early as 1978, results from the Harvard Cooperative Stroke Registry (HCS Registry) showed that one-third of stroke patients had a reported episode of AF. For the first time, then, the disease was clearly defined as an important factor in the development of embolic brain accidents [2]. The same year saw the release of the first Framingham Heart Study data to clearly demonstrate the role of AF in thromboembolic events: stroke risk increased 5.6-fold in AF patients without known valve defects [3]. Today, forty years later, the accumulated evidence on the topic is overwhelming and indisputable. At the same time, there is undisputed evidence that incidence, morbidity, and affected population are significantly different for the different types of AF. This quite logically raises the question of possible differences in the thromboembolic potential of paroxysmal and non-paroxysmal (persistent and permanent) AF. However, most studies address the problem for the entire population of AF patients. There is no clarity on the degree of thromboembolic risk depending on AF duration. The purpose of this material is to critically analyze existing data on the subject.

\footnotetext{
* Corresponding author: Negreva Mariya Negrinova
} 


\section{Methods of science research}

A systematic overview of all epidemiological studies concerning thromboembolic risk in AF presented in MEDLINE and PubMed databases was conducted. Emphasis was placed on thromboembolic potential as a function of disease duration and AF type, according to its duration - paroxysmal and non-paroxysmal (persistent and permanent). We used the following keywords: (non) paroxysmal, persistent and permanent AF, AF type, epidemiology, prognosis, mortality, anticoagulant and antiplatelet therapy. All data for the period 2005-2019 were analyzed. This study included studies with a clear protocol and definition of AF, reliable sources of medical records, clinical assessment methods, and followup of relevant patient subgroups.

\section{Incidence, morbidity and clinical characteristics of AF. Differences according to type}

In 2009, in a review by Aronow and Banach, $\mathrm{AF}$ was declared the "new epidemic of the aging world", and is still regarded as such today [4]. This is due to the results of large epidemiological studies that show an almost double incidence of the disease over a ten-year period, currently reaching up to $3 \%$ of the total population [5]. It varies significantly according to age: $0.12 \%-0.16 \%$ for the population under $49 ; 3.7 \%-4.2 \%$ for the $60-70$ age group and $10 \%-17 \%$ for the population over 80 years. It is more common in men, with a ratio of 1.2 to 1 .

Both current and predicted epidemiological data for the next few decades are alarming. In Europe, the number of people affected by the disease is expected to increase steadily, reaching 17 million people in 2060 and up to 215,000 newly diagnosed cases annually [6]. The most significant increase is expected in age group over 75.

The same alarming trend is predicted worldwide [7]. Probable cause is the aging of the population, as well as improved diagnostic and therapeutic approaches to patients with cardiovascular diseases.

It is also believed that the results so far significantly underestimate the real situation and the incidence is much higher. The well-established fact is that a small number of cases (15\%-46\%) occur asymptomatically and are diagnosed accidentally or during an embolic incident [8]. Therefore, active screening for signs of the disease would result in significantly higher values.

Studies of outpatient and hospitalized patients show that permanent AF is the most commonly diagnosed form of the disease, affecting $40 \%-50 \%$ of AF patients, the remaining cases being split almost equally between the paroxysmal and persistent form of the disease [5].

Paroxysmal AF manifests its specific characteristics. It occurs generally in younger patients (decades 40-49, 50-59, and 60-69 years), who have less concomitant heart and extra-cardiac diseases. It is difficult to determine its actual incidence. Episodes can be very short (just above $30 \mathrm{sec}$ ), run asymptomatically, and are more often ignored by the patients themselves. According to some epidemiological studies, paroxysmal AF is more often asymptomatic than other AF types - up to $81 \%$ of cases [9]. These specific clinical features cause PAF to be frequently undiagnosed and underestimated [10]. The risk of skipping anticoagulant therapy in PAF is the greatest.

Different AF types are associated not only with different patient characteristics and profiles, but also with different longterm prognosis. According to studies conducted in France and Canada, the rate of progression of paroxysmal to permanent AF during the first year is about 9\%, and by the fifth year it reaches 18-25\%. Persistent AF has a significantly higher incidence [11]. According to the Euro Heart Survey data, progression to permanent AF is reported in $15 \%$ of paroxysmal patients and $30 \%$ of patients with persistent form of the disease [12].

\subsection{Thromboembolic risk according to AF type}

Atrial fibrillation is one of the ten potentially modifiable factors associated with the clinical manifestation of acute stroke [13]. In the early 1990s, the first results of the Framingham Heart Study project identified the disease as a risk factor for stroke [14]. Later, epidemiological studies showed that its presence can increase the risk of stroke by up to $20 \%$ per year, with a significant number of risk factors having a modulating effect: age, hypertension, diabetes mellitus, previous thromboembolic events, peripheral vascular disease, HF and female sex [15].

The high thromboembolic potential that the AF population carries as a whole, and at the same time its pronounced clinical heterogeneity, are prerequisites for a search for differences in embolic risk among AF subpopulations, in particular between the paroxysmal and the non-paroxysmal (including persistent and persistent) forms. 
The first studies and meta-analyzes on the subject were published at the end of the last century and the beginning of the present. The results did not establish a relationship between AF type and disease prognosis, incl. incidence of thromboembolic events and mortality. For example, in a population of aspirin-treated patients, Hart et al. found no difference in the incidence of embolic incidents between the intermittent and permanent form of the disease [16]. The authors themselves, however, clearly outline the main weaknesses of the study, namely its limitation only among hospitalized population, which is unlikely to be representative of the general AF population. Moreover, the frequency and duration of the episodes were not documented correctly enough.

A sub-analysis of the ACTIVE W study, published by Hohnloser et al., presented similar results: no statistically significant difference in the embolic potential of the paroxysmal and prolonged form of the disease [17]. Over 6,000 patients undergoing oral anticoagulation or combination therapy with clopidogrel and aspirin were studied. Although 7 years have passed since the study by Hart et al., ACTIVE W sub-analysis was the first to follow, with the observed population again not being "pure" with respect to antithrombotic drugs. The systematic review, suggested by the atrial fibrillation working group again did not present the type of AF as an independent risk factor for the incidence of embolic incidents [18]. A year later, similar data were presented by Hughes et Lip [19]. Results from the Danish Cohort were published in 2010 [20]. Paroxysmal AF patients were subject of the study, since the authors considered the knowledge in this area to be too limited. The incidence of ischemic stroke was found to be similar in paroxysmal and permanent AF (26 versus 29 cases / 1000 patient years).

The Euro Heart Survey involving over 3000 AF patients monitored for one year found a significantly higher incidence of ischemic transient attack in the persistent form of the disease (30 versus 9 cases, $p<0.001$ ) and no significant difference in the number of ischemic strokes (22 versus 19 cases, $p=0.58$ ) [21]. Lip et al. found a lower incidence of ischemic strokes in the paroxysmal form of AF compared to persistent $0.93 \%$ versus $1.73 \%$ ischemic strokes/systemic embolic events per year), with its "advantage" disappearing in the "high-risk" subgroup (1.27\% versus $2.08 \%$ for the paroxysmal and persistent form, respectively) [22].

A large retrospective study by Banerjee et al. on more than 7000 patients also presented results in support of the abovementioned studies [23]. Stroke risk in patients with paroxysmal, persistent, and permanent AF was similar and independent of arrhythmia duration. Similar results have been found in other large studies that suggested that AF type was not relevant to its embolic risk [23, 24].

Of particular interest are the results of the GISSI-AF study, performed on 771 patients with paroxysmal AF and 463 patients with persistent $\mathrm{AF}$, monitored for a period of one year. The researchers found no difference in the incidence of thromboembolic events in persistent and paroxysmal AF [25]. However, disease recurrences reported non-invasively (frequent clinical visits and transtelephonic monitoring devices) were associated with a significant increase in the incidence of thromboembolic events $(0.5 \%$ in patients without recurrences versus $1.74 \%, 1.28 \%$ and $1.18 \%$, registered in symptomatic, asymptomatic or both types of recurrences, respectively). The differences found were independent of intake of oral anticoagulants and CHADS2 score (HR 2.93; CI 95\%; 0.8-10.9; $\mathrm{p}=0.11$ ).

Despite the alarming data on the increasing incidence of the disease, studies to date on thromboembolic risk in different types of AF remain scarce, as the abovementioned data show. The small number of studies and meta-analyzes presented during this period do not really address the problem. They focus mainly on clinical, laboratory and ultrasound indicators as possible predictors of embolic incidents and prognosis in patients with AF [26-29]. A possible reason for this is lack of data on AF type at patient discharge.

Of particular interest is the recently published meta-analysis by Ganesan et al. because of the analyzed studies and obtained results [30]. It included only prospective studies: randomized controlled, cohort, and case series describing the prognosis depending on the type of $\mathrm{AF}$, which is a serious prerequisite for the reliability of the results. In total, they were twelve studies for the period from 1990 to 2015 on 99996 patients. A higher risk of non-paroxysmal AF was found compared to paroxysmal with inadequate oral anticoagulant protection. The total hazard ratio (HR) for thromboembolism in non-paroxysmal AF relative to paroxysmal AF was 1.384 (95\% CI: 1.191-1.608, p < 0.001). HR for overall mortality was 1.217 (95\% CI: $1.085-1.365, \mathrm{p}<0.001)$. The non-paroxysmal form of the disease was associated with a significant increase in thromboembolism and mortality, which supports the introduction of new drug therapies that prevent the progression and chronification of arrhythmia. Moreover, the meta-analysis data demonstrate the existence of a comorbidity-independent effect of AF type on mortality and thromboembolism, while lacking a correlation between the hemorrhagic risk, associated with comorbidities and AF type. The reported increase in the risk of thromboembolism and mortality persists even after standardization in regard to stroke risk factors. Thromboembolic risk and mortality were higher in the patients without oral anticoagulation but remained high in the oral anticoagulant group. An important clarification is that the distribution of AF clinical subtypes was performed according to the 2006 
AHA/ACC/ESC guidelines. It is important to note that the authors of the meta-analysis did not answer the question of the degree of independence of the effect of total AF duration on stroke risk in patients with paroxysmal AF in whom anticoagulant therapy for stroke prevention is debatable.

A significantly higher incidence of embolic events and mortality in the non-paroxysmal form of AF has been also reported by other authors. The J-RHYTHM Registry examined 7,406 patients monitored for two years in order to perform a comparative analysis of thromboembolic risk between the three major nonvalvular AF subtypes [31]. The ratio of patients on anticoagulant prophylaxis at baseline were 78.6\%, 90.0\%, and 91.8\% with paroxysmal, persistent, and chronic AF, respectively. A total of 126 thromboembolic incidents were reported. The incidence was twice as high in patients with permanent AF (2.29\%), compared to patients with paroxysmal (1.16\%) or persistent AF (1.20\%) $(\mathrm{p}<0.001)$. However, after control in the analysis for oral anticoagulants and CHA2DS2-VASc, no significant differences in thromboembolic risk were detected, depending on the type of AF (paroxysmal AF as a reference category and persistent $\mathrm{AF}$ ).

Some studies have shown exactly the opposite - a statistically significantly higher incidence of embolic cerebrovascular incidents in paroxysmal atrial fibrillation [32]. The intermittently organized atrial contraction after periods of AF is accepted as the main explanation for this.

This contradiction in the results that gave reason to Go et al. to observe thromboembolic incidents in AF in a slightly different light, emphasizing the paroxysmal form of the disease, since they consider it to be the "challenge" in the decision to carry out thromboprophylaxis [33]. For them, AF duration is undoubtedly associated with its thromboembolic potential. However, they do not place it in the familiar framework of paroxysmal and non-paroxysmal AF, but use the concept of burden of AF (the amount of time spent in atrial fibrillation). They studied a total of 25,268 non-anticoagulated patients and performed continuous outpatient non-invasive ECG monitoring for a 14-day period. The group was monitored for ischemic strokes and other arterial thromboembolic events. Thromboembolic risk was assessed in the absence of anticoagulant protection. The burden of AF was defined as percentage of the duration compared to the total monitoring time. The number of patients with paroxysmal AF was 1965 and the incidence of thromboembolic events was 29 . The recorded mean AF burden was $4.4 \%$. Higher burden $(\geq 11 \%)$ was associated with higher ischemic risk, regardless of known risk factors. Thromboembolic risk remained three times higher even after standardization for ATRIA (Anticoagulation and Risk Factors in Atrial Fibrillation) or CHA2DS2-VASc risk sum (HR = 3.13 and 3.16). This led the authors to believe that the total "burden of AF" in a particular patient could be a useful indicator in deciding on anticoagulant prevention of ischemic stroke.

Previous analyzes, however, examining predominantly anticoagulated populations with implanted electronic devices (e.g. dual chamber pacemaker, implantable cardioverter-defibrillator, or a cardiac resynchronization therapy device) also identified a relationship between "total recorded time spent in AF" and reported embolic risk. Summary data from TRENDS, PANORAMA and the Italian Clinical Service Project on over 10,000 patients showed that increase in AF duration leads to increase in stroke risk (HR 1.03 per hour; 95\% CI, 1.00-1.05) [34]. In the ASSERT study, asymptomatic atrial tachycardia detected by the implanted devices lasting $>6$ min over the last 3 months correlated with a higher incidence of ischemic stroke or systemic embolism (HR, 2.49; 95\% CI, 1.28-4.85) [35]. However, as the authors themselves point out, there were no ECG records of AF paroxysms in the studied patients, but only the results presented by the implanted devices. In this sense, the data presented by Turakhia et al., are extremely valuable [36]. Of the 9850 monitored patients with proven AF paroxysms, 187 were registered with ischemic stroke or systemic embolism. A daily AF burden of 5.5 hours, recorded up to 30 days before the incident, was associated with an increased risk of stroke, regardless of whether the patient was receiving anticoagulation therapy.

Our understanding of the thromboembolic complications in AF and the risk factors associated with them are based mainly on studies that identify AF as a binary-only phenomenon (i.e., absence or presence of a rhythmic disturbance), placed within the artificially created restriction frame of paroxysmal and non-paroxysmal form. They do not consider the presence of a rhythmic disorder as a quantitative phenomenon, which, over time, leads to a qualitatively new clinical manifestation - that of thromboembolic events. However, as is well known, the natural course of AF is characterized by recurrence and often chronification. In this sense, "AF burden" as a concept, representing the amount of AF over a period of time, is a natural characteristic of the disease, dynamically changing with it and dynamically reflecting its clinical nature.

With a conscious understanding of this, the American Heart Association has issued a formal opinion on "AF burden". Despite the accumulated evidence from recent years that greater AF burden is associated with higher embologenic risk, there is still no clear definition of the threshold for this burden, which determines cerebrovascular accidents and requires anticoagulant treatment [37]. Lack of knowledge and a clearly fixed 'embologenic threshold of AF burden' are 
the most likely reasons for the conflicting results regarding thromboembolic risk in different types of AF. An important point in the AF/embolic potential relationship is the usual presence of risk factors, which in themselves have a prothrombotic effect. We can predict, but we cannot establish for sure their significance for a particular patient, since they themselves also have a temporal characteristic and manifestation. All this further complicates the application of clear and sharp demarcation frameworks such as paroxysmal/non-paroxysmal AF.

Moreover, frequent asymptomatic episodes of the disease make subjective (anamnestic) assessment an unreliable method for determining the presence and, respectively, AF type. The question naturally arises of biomarkers that could reflect the presence/burden of $\mathrm{AF}$ and allow the assessment of embolic risk. Given the well-established fact that embolic incidents in AF are associated with changes in hemostatic balance, it seems logical to look for biomarkers among hemostatic indicators. In addition to the established scales for embologenic risk in AF, they could optimize the choice of antithrombotic prophylaxis, especially in low-risk patients for whom it is being increasingly established that embolic incidence is not as low.

In this regard, we conducted our clinical study, which found that even a small burden of PAF (8.14 \pm 0.76 hours) was associated with significant changes in major indicators of fibrinolysis (Table 1) [38].

Table 1 Fibrinolytic markers in patients with PAF and controls in sinus rhythm (results from our previous study).

\begin{tabular}{llll}
\hline Fibrinolytic markers & Patients & Controls & P values \\
\hline Plasminogen levels (\%) & $159.40 \pm 4.81$ & $100.2 \pm 2.88$ & $\mathrm{p}<0.001$ \\
t-PA level (ng/mL) & $11.25 \pm 0.35$ & $6.05 \pm 0.31$ & $\mathrm{p}<0.001$ \\
PAI-1 activity (AU/mL) & $7.33 \pm 0.37$ & $15.15 \pm 0.52$ & $\mathrm{p}<0.001$ \\
$\alpha 2$-antiplasmin activity (\%) & $112.9 \pm 2.80$ & $125.60 \pm 3.74$ & $\mathrm{p}<0.05$ \\
Vitronectin (mcg/mL) & $134.7 \pm 5.83$ & $287.3 \pm 10.44$ & $\mathrm{p}<0.001$ \\
D-dimer (mg/L) & $0.53 \pm 0.07$ & $0.33 \pm 0.02$ & $\mathrm{p}<0.05$ \\
\hline
\end{tabular}

The summary analysis of the results led us to conclude that short episodes of the disease (duration $<24$ hours) are associated with increased fibrinolytic activity due to activated coagulation. The logistic and ROC curve analysis showed that vitronectin values were the most reliable indicator to reject the presence of PAF, while the D-dimer had the lowest diagnostic value. (Table 2, Figure 1).

Table 2 Estimators of the parameters of univariate logistics models for association between fibrinolytic factors and the absence of PAF manifestation.

\begin{tabular}{llllll}
\hline Factors & $\beta_{0}$ & $\beta_{1}$ & $\mathbf{x}^{*}$ & Odds ratio & Accuracy (\%) \\
& $\mathbf{p}$-value & $\mathbf{p}$-value & & & \\
\hline Plasminogen level & 10.354 & -0.083 & 124.75 & 17.875 & 80.58 \\
& $<0.001$ & $<0.001$ & & & \\
t-PA level & 8.835 & -1.034 & 8.54 & 12.114 & 77.67 \\
& $<0.001$ & $<0.001$ & & & \\
PAI-1 activity & -9.450 & 0.885 & 10.68 & 34.554 & 85.44 \\
& $<0.001$ & $<0.001$ & & & \\
$\alpha 2 \quad$ antiplasmin & -2.772 & 0.023 & 120.52 & 4.042 & 66.67 \\
activity & $<0.013$ & 0.011 & & & \\
D-dimer & 0.753 & -1.806 & 0.42 & 2.236 & 57.84 \\
& $<0.029$ & $<0.015$ & & & \\
Vitronectin & -11.04 & 0.0574 & 196.30 & 76.444 & 88.35 \\
& $<0.001$ & $<0.001$ & & & \\
\hline
\end{tabular}




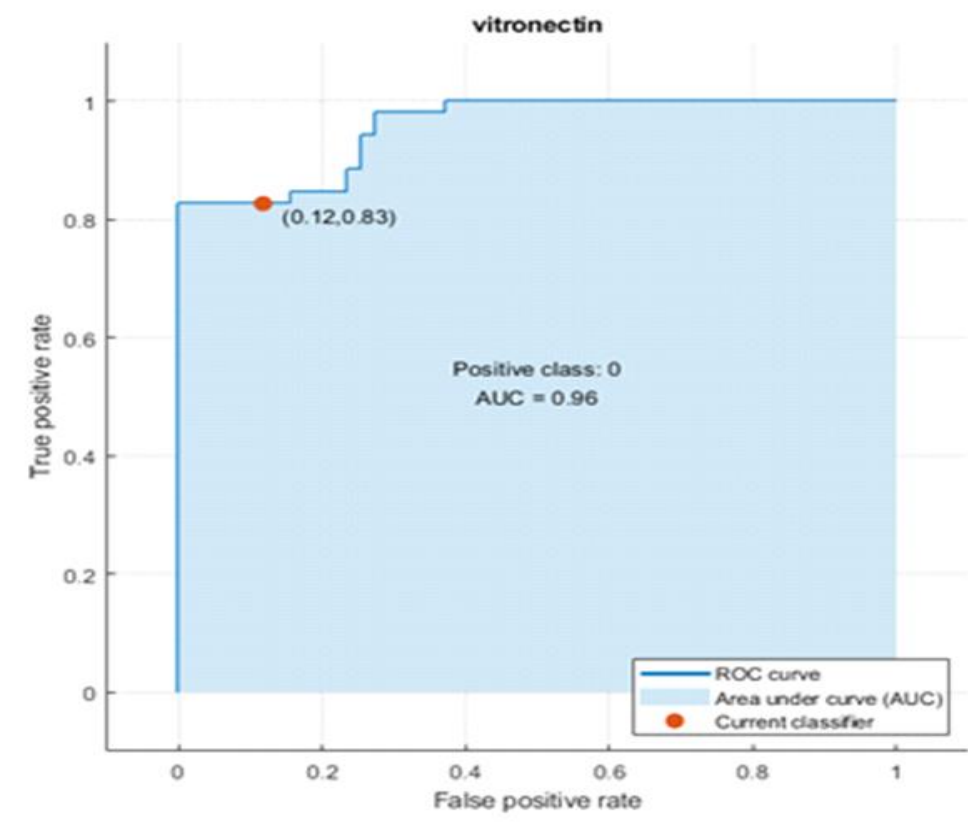

Figure 1 ROC curve for the diagnostic value of vitronectin.

\section{Conclusion}

The results of the studies conducted to date on thromboembolic risk in AF are controversial. There is no consensus on its stratification connected with the duration of the disease (paroxysmal/non-paroxysmal AF type). According to present clinical guidelines, anticoagulant therapy or thromboembolic risk, respectively, are independent of the AF type (paroxysmal/non-paroxysmal). However, the fact that AF has dynamic nature and its paroxysmal form often progresses to non-paroxysmal cannot be overlooked. Further studies in search of the embologenic threshold of AF and biomarkers for its diagnosis are necessary for optimization of anticoagulant prophylaxis.

\section{Compliance with ethical standards}

\section{Disclosure of conflict of interest}

The authors have no conflicts of interest to declare.

\section{References}

[1] Fuster V, Ryden LE, Cannom DS, et al. (2006). ACC/AHA/ESC guidelines for the management of patients with atrial fibrillation: a report of the American College of Cardiology/American Heart Association Task Force on Practice Guidelines and the European Society of Cardiology Committee for Practice Guidelines. Europace, 8, 651745.

[2] Mohr JP, Caplan LR, Melski JW et al. (1978). The Harvard Cooperative Stroke Registry: a prospective registry. Neurology, 28(8), 754-62.

[3] Wolf PA, Dawber TR, Thomas HE Jr et al. (1978). Epidemiologic assessment of chronic atrial fibrillation and risk of stroke: the Framingham study. Neurology, 28, 973-77.

[4] Aronow WS and Banach M. (2009). Atrial Fibrillation: The New Epidemic of the Ageing World. J Atr Fibrillation, 1(6), 154.

[5] Zoni-Berisso M, Lercari F, Carazza T et al. (2014). Epidemiology of atrial fibrillation: European perspective. Clin Epidemiol, 6, 213-20.

[6] Krijthe BP, Kunst A, Benjamin EJ et al. (2013). Projections of the number of individuals with atrial fibrillation in the European Union from 2000 to 2060. Eur Heart J, 34, 2746-51. 
[7] Zulkifly H, Lip GYH and Lane DA. (2018). Epidemiology of atrial fibrillation. Int J Clin Pract, 72(3), e13070.

[8] Streur M, Ratcliffe SJ, Ball J et al. (2017).Symptom Clusters in Adults With Chronic Atrial Fibrillation. J Cardiovasc Nurs, 32(3), 296-303.

[9] Quirino G, Giammaria M, Corbucci G et al. (2009). Diagnosis of paroxysmal atrial fibrillation in patients with implanted pacemakers: relationship to symptoms and other variables. Pacing Clin Electrophysiol, 32(1), 91-8.

[10] Primo J, Gonçalves H, Macedo A et al. (2017). Prevalence of paroxysmal atrial fibrillation in a population assessed by continuous 24-hour monitoring. Rev Port Cardiol, 36(7-8), 535-46.

[11] DE Sisti A, Leclercq JF, Halimi F et al. (2014). Evaluation of time course predicting factors of progression of paroxysmal or persistent atrial fibrillation to permanent atrial fibrillation. Pacing Clin Electrophysiol, 37, 345355.

[12] Jahangir A, Lee V, Friedman PA et al. (2007). Long-term progression and outcomes with aging in patients with lone atrial fibrillation, A 30-year follow up study. Circulation, 115, 3050-56.

[13] O'Donnell MJ, Chin SL, Rangarajan S et al. (2016). Global and regional effects of potentially modifiable risk factors associated with acute stroke in 32 countries (INTERSTROKE): A case-control study. Lancet, 388, 761-75.

[14] Benjamin EJ, Levy D, Vaziri SM et al. (1994). Independent risk factors for atrial fibrillation in a population-based cohort. The Framingham Heart Study. JAMA, 271(11), 840-4.

[15] Furie KL, Goldstein LB, Albers GW et al. (2012). Oral antithrombotic agents for the prevention of stroke in nonvalvular atrial fibrillation: a science advisory for healthcare professionals from the American Heart Association/American Stroke Association. Stroke, 43(12), 3442-53.

[16] Hart RG, Pearce LA, Rothbart RM et al. (2000). Stroke with intermittent atrial fibrillation: incidence and predictors during aspirin therapy. J Am Coll Cardiol, 35, 183-87.

[17] Hohnloser SH, Pajitnev D, Pogue J et al. (2007). Incidence of stroke in paroxysmal versus sustained atrial fibrillation in patients taking oral anticoagulation or combined antiplatelet therapy: an ACTIVE W Substudy. J Am Coll Cardiol, 50, 2156-61.

[18] The Stroke Risk in Atrial Fibrillation Working Group. (2007). Independent predictors of stroke in patients with atrial fibrillation: a systematic review. Neurology, 69, 546-54.

[19] Hughes M and Lip GY. (2008). Stroke and thromboembolism in atrial fibrillation: a systematic review of stroke risk factors, risk stratification schema and cost effectiveness data. Thromb Haemost, 99, 295-304.

[20] Friberg L, Hammar N and Rosenqvist M. (2010). Stroke in paroxysmal atrial fibrillation: report from the Stockholm Cohort of Atrial Fibrillation. Eur Heart J, 31(8), 967-75.

[21] Nieuwlaat R, Prins MH, Le Heuzey JY et al. (2008). Prognosis, disease progression, and treatment of atrial fibrillation patients during 1 year: follow-up of the Euro Heart Survey on atrial fibrillation. Eur Heart J, 29(9), 1181-9.

[22] Lip GYH, Frison L and Grind M. (2008). Stroke event rates in anticoagulated patients with paroxysmal atrial fibrillation. J Intern Med, 264, 50-61.

[23] Banerjee A, Taillandier S, Olesen JB et al. (2013). Pattern of atrial fibrillation and risk of outcomes: the Loire Valley Atrial Fibrillation Project. Int J Cardiol, 167(6), 2682-7.

[24] Inoue H, Atarashi H, Okumura K et al. (2014). Thromboembolic events in paroxysmal vs. permanent non-valvular atrial fibrillation. Subanalysis of the J-RHYTHM Registry. Circ J, 78(10), 2388-93.

[25] Disertori M, Franzosi MG, Barlera S et al. (2013). GISSI-AF investigators. Thromboembolic event rate in paroxysmal and persistent atrial fibrillation: data from the GISSI-AF trial. BMC Cardiovasc Disord, 13, 28.

[26] Pisters R, Lane DA and Marin AF. (2012). Stroke and thromboembolism in atrial fibrillation. Circ J, 76, 22892304.

[27] Gage BF, Waterman AD, Shannon W et al. (2001). Validation of clinical classification schemes for predicting stroke: results from the National Registry of Atrial Fibrillation. JAMA, 285, 2864-2870.

[28] Lip GY, Nieuwlaat R, Pisters R et al. (2010). Refining clinical risk stratification for predicting stroke and thromboembolism in atrial fibrillation using a novel risk factor-based approach. Chest, 137, 263-272. 
[29] Singer DE, Chang Y, Borowsky LH et al. (2013). A new risk scheme to predict ischemic stroke and otherthromboembolism in atrial fibrillation: the ATRIA study stroke risk score. J Am Heart Assoc, 2, e000250.

[30] Ganesan AN, Chew DP, Hartshorne T et al. (2016). The impact of atrial fibrillation type on the risk of thromboembolism, mortality, and bleeding: a systematic review and meta-analysis. Eur Heart J, 21, 37(20), 1591602 .

[31] Inoue H, Atarashi H, Okumura K et al. (2014). For the J-RHYTHM Registry Investigators. Thromboembolic Events in Paroxysmal vs. Permanent Non-Valvular Atrial Fibrillation- Subanalysis of the J-RHYTHM Registry. Circ J, 78, 2388-2393.

[32] Primo J, Gonçalves H, Macedo A et al. (2017). Prevalence of paroxysmal atrial fibrillation in a population assessed by continuous 24-hour monitoring. Rev Port Cardiol, 36(7-8), 535-546.

[33] Go AS, Reynolds K, Yang J et al. (2018). Association of Burden of Atrial Fibrillation With Risk of Ischemic Stroke in Adults With Paroxysmal Atrial Fibrillation: The KP-RHYTHM Study. JAMA Cardiol, 3(7), 601-608.

[34] Boriani G, Glotzer TV, Santini M et al. (2014). Device-detected atrial fibrillation and risk for stroke: an analysis of $>10,000$ patients from the SOS AF project (Stroke preventiOn Strategies based on Atrial Fibrillation information from implanted devices). Eur Heart J, 35(8), 508-516.

[35] Healey JS, Connolly SJ, Gold MR et al. (2012). ASSERT Investigators. Subclinical atrial fibrillation and the risk of stroke. N Engl J Med, 366(2), 120-129.

[36] Turakhia MP, Ziegler PD, Schmitt SK et al. (2015). Atrial Fibrillation Burden and Short-Term Risk of Stroke: CaseCrossover Analysis of Continuously Recorded Heart Rhythm From Cardiac Electronic Implanted Devices. Circ Arrhythm Electrophysiol, 8(5), 1040-7.

[37] Chen LY, Chung MK, Allen LA et al. (2018). American Heart Association Council on Clinical Cardiology; Council on Cardiovascular and Stroke Nursing; Council on Quality of Care and Outcomes Research; and Stroke Council. Atrial Fibrillation Burden: Moving Beyond Atrial Fibrillation as a Binary Entity: A Scientific Statement From the American Heart Association. Circulation, 137(20), e623-e644.

[38] Negreva M, Georgiev S and Vitlianova K. (2016). Early effects of paroxysmal atrial fibrillation on plasma markers of fibrinolysis. Medicine (Baltimore), 95(45), e5184.

\section{How to cite this article}

Vitlianova KD, Negreva MN and Madjova CR. (2020). Paroxysmal and non-paroxysmal atrial fibrillation: does the arrhythmia type influence thromboembolic risk? World Journal of Advanced Research and Reviews, 6(1), 192-199. 